Vol. 5, n 1 | 2001

Varia

\title{
La voie de Paolo Zacchias : médecine et crime
}

\section{Michel Porret}

\section{OpenEdition}

Journals

Édition électronique

URL : https://journals.openedition.org/chs/786

DOI : $10.4000 /$ chs.786

ISSN : 1663-4837

Éditeur

Librairie Droz

\section{Édition imprimée}

Date de publication : 1 janvier 2001

Pagination : 129-133

ISBN : 2-600-00607-9

ISSN : 1422-0857

Référence électronique

Michel Porret, "La voie de Paolo Zacchias : médecine et crime », Crime, Histoire \& Sociétés / Crime,

History \& Societies [En ligne], Vol. 5, $n^{\circ} 1$ | 2001, mis en ligne le 02 avril 2009, consulté le 23 mars 2022

URL : http://journals.openedition.org/chs/786 ; DOI : https://doi.org/10.4000/chs.786 


\title{
La voie de Paolo Zacchias: médecine et crime
}

\author{
Michel Porret
}

Alessandro PASTORE, Il medico in tribunale. La perizia medica nella procedura penale d'antico regime (secoli XVI-XVIII), Biblioteca dell'AST (Archivio Storico Ticinese, No2), Bellinzona, Edizioni Casagrande, 1998, 251 p., couverture illustrée, 16 figures hors texte, tableaux, 2 cartes géographiques, index, bibliographie dans les notes, ISBN 88-7713-268-X

Élégant professeur d'histoire moderne à 1'Université de Vérone et spécialiste des institutions hospitalières de l'Ancien régime, Alessandro Pastore signait en 1991 une belle étude comparative consacrée au problème dramatique de la criminalité en temps de peste dans l'Europe moderne (Crimine et giustizia in tempo di peste nell'Europa moderna, Laterza, Rome-Bari, 236 p.) Attendant toujours sa traduction française, cette importante enquête sur les sensibilités modernes montrait notamment la manière dont la violence interpersonnelle culminait durant les épidémies qui décimaient les communautés traditionnelles et ruinaient les mécanismes du contrôle social. Revenant à la charge sur les problèmes de l'anomie sociale particuliers à l'Ancien régime, le moderniste de Vérone nous donne à lire aujourd'hui un ouvrage de belle facture éditoriale, très réussi sur un objet neuf: l'impact des expertises médico-légales dans les tribunaux criminels (et civils) ayant leur siège en Italie du Nord, en Toscane, en Vénétie, et dans la Suisse lombarde.

«L'historien de la médecine légale commence sa propre enquête par l'étude des lois appliquées aux cas concrets de la vie sociale et en déduit des principes généraux desquels il est possible de comprendre la genèse et le développement du savoir médical lié au droit ». Ces conclusions programmatiques à la journée d'étude consacrée en mai 1983 à Reggio Emilia aux problèmes historiques de la médecine légale (sources, herméneutique, historicité, modèles antiques, médiévaux, modernes et contemporains) montrent l'enjeu de cette discipline dans la perspective d'Alessandro Pastore sur le plan de l'histoire sociale, de celle des institutions pénales, de celle des transgressions et des normes'. Or, l'histoire de la médecine légale a longtemps fasciné les légistes eux-mêmes: attachés à l'idée du progrès médical, ils en devenaient les historiens, parfois peu critiques, comme pouvaient l'être les juristes qui écrivaient l'histoire institutionnelle ou anecdotique de Thémis. Pour s'en convaincre, il suffit de parcourir certains ouvrages d'Antoine Lacassagne (Précis de Médecine Judiciaire, Paris, 1878, «Historique», pp. 1-19), ou de relire

Centro italiano di storia sanitaria e ospitaliera di Reggio Emilia, La storia della medicina legale, ricerche e problemi. Atti della giomata di studio (Reggio Emilia, 21 maggio 1983), a cura di Cosimo Damiano Fonseca, Reggio, 1987, pp. 15-40 (loc. cit. p. 39). 
l'ouvrage pionnier du légiste lyonnais Edmond Locard: Le XVII $I^{\text {eme }}$ siècle médicojudiciaire, Lyon, 1902, mis sous la double autorité des Lumières et du progrès scientifique («Préface», pp. i-xiv).

Comme le montre l'ouvrage d'Alessandro Pastore, aujourd'hui, la médecine légale est devenue un objet d'étude qui intéresse les historiens du corps, de la justice pénale ou civile et des institutions ou des savoirs qu'elle légitime. Ils y appliquent les règles de leur discipline: établissement, critique interne et externe des sources, reconstruction du contexte institutionnel, juridique et social, analyse des traditions médicales et des catégories professionnelles, mise en place d'un questionnaire qui donne sens à l'expertise médico-légale telle qu'elle s'affirme en Europe moderne dès le XVI ${ }^{\text {eme }}$ siècle, notamment dans les régions labourées par la renaissance du jus romanum. Vaste programme qui permet d'historiciser les objets et les problématiques de la médecine légale: peut-on encore écrire une histoire du corps moderne en ignorant ses conceptions matérialistes élaborées par les usages et les doctrines médico-légales dès la Renaissance? Peut-on comprendre la formidable «révolution anatomique» imposée par Vésale à la Renaissance en négligeant la pratique croissante de la dissection des cadavres de suppliciés dans les théâtres publics d'anatomie? (Andrea Carlino, La fabbrica del corpo. Libri e dissezione nel Rinascimento, Turin, Einaudi, 1994). L'histoire de la médecine légale s'enracine dans celle du traitement moderne du corps, de l'anomie sociale et des savoirs sur ces objets.

Soulignons que la place sociale des pratiques médico-légales dans un monde alors écrasé par la religion est complexe. En effet, bien que la médecine légale implique une conception matérialiste des usages et des doctrines du corps (pensons à l'autopsie), sa genèse moderne repose pourtant sur une problématique religieuse: stigmates, possessions, miracles, guérisons «magiques». À la Renaissance, le père fondateur de la médecine légale, Paolo Zacchias (1584-1659, Quaestiones medicolegales, 1621-1635), attaché au tribunal de Rote, veut distinguer les maladies vraies des «maladies simulées», enquête sur les «guérisons miraculeuses», diagnostique la «folie», examine aussi la véracité des miracles qui marquent les hommes et les choses $^{2}$.

En montrant la manière dont la culture médico-légale devient un élément constitutif de la pratique juridique des juges italiens pour qualifier un délit afin de motiver une peine harmonisée sur l'atrocité d'un crime de sang, Alessandro Pastore étudie la problématique de la modernité judiciaire de l'époque moderne autour des figures contrastées de l'homo criminalis et de la victime. En outre, il s'inscrit dans un champ historiographique relativement vierge: pour mémoire, et en ce qui concerne l'Ancien Régime, évoquons la somme de l'historienne suisse Esther FisherHomberger (Medizin vor Gericht. Gerichtsmedizin von der Renaissance bis zur Aufklärung, Berne (etc.), Hans Buber, 1983, 487 p.), ainsi que l'étude collective publiée par Michael Clark et Catherine Crawford (Legal Medicine in History, Cambridge, CUP, 1994, 364 p.). D'un côté, une érudite histoire des idées médicolégales en Occident; de l'autre, des monographies sur la dimension sociale de cette discipline devenue dès la Renaissance l'auxiliaire des juges du siège ${ }^{3}$.

2 Cf. Charles Vallon, Georges Genil-Perin, La psychiatrie médico-légale dans l'auvre de Zacchias (1584-1659), Paris, 1912.

3 On ajoutera à ces ouvrages récents, des travaux plus anciens mais parfois encore utiles (érudition, anecdotes, sociologie des légistes, etc.): Charles Desmaze, Histoire de la médecine légale en France 
$\mathrm{Au} \mathrm{XIX}^{\mathrm{ème}}$ siècle, forts du code pénal et du positivisme de leur temps, les magistrats ne pourront plus se passer des médecins légistes et de leur savoir «objectif» pour mieux qualifier les «circonstances» morales et matérielles des crimes de sang ou de la folie comme vient de le démontrer avec une grande pertinence et dans une langue limpide Frédéric Chauvaud (Les experts du crime. La médecine légale en France au XIX ${ }^{\text {ème }}$ siècle, Paris, Aubier, 2000,300 p.), en valorisant fortement la rupture entre l'Ancien Régime et le XIX ${ }^{\text {eme }}$ siècle en ce qui concerne la modernité de l'expertise médico-légale. Par l'étude minutieuse du «savoir-faire» des légistes français, alliés ou adversaires des avocats, Chauvaud mesure la triomphante institutionnalisation de la médecine légale devant les cours criminelles. Il souligne l'impact normatif et judiciaire de la «preuve expertale» dans la connaissance et l'analyse de l'anomie sociale: ainsi, sur la scène du crime, dans leurs laboratoires et devant la cour et les jurys, les légistes positivistes poursuivent l'ouvre des empiristes du siècle des Lumières, eux-mêmes héritiers des «experts » de la Renaissance.

En 1988 déjà, les historiennes anglaises Anne Crowther et Brenda White publiaient une remarquable monographie (injustement méconnue par I'historiographie) concernant l'enracinement institutionnel de la médecine légale clinique et universitaire à Glasgow entre 1839 et le début des années 1960 (On Soul and Conscience, the Medical Expert and Crime. 1500 Years of Forensic Medecine in Glasgow, Aberdeen, Aberdeen University Press, 1988, 150 p.). De même qu'en France, mais dépendante du développement précoce de la police judiciaire anglaise, la médecine légale écossaise liait fortement le traitement judiciaire de la criminalité à la connaissance de ses composantes «morales» et matérielles telles que l'avaient parfois élaborées les magistrats et les médecins du XVIII ${ }^{\text {eme }}$ siècle.

Divisé en huit grandes parties et en une quarantaine de brefs et précis chapitres qu'annonce une « introduction » définissant le rôle et la place croissante de la médecine légale dans la tradition criminologique et pénale occidentale jusqu'à aujourd'hui, l'ouvrage de Pastore est écrit au plus près des sources médico-légales: les procès criminels de l'époque moderne, notamment les dossiers du tribunal du «Torrone» de Bologne (littéralement «Tribunal du Donjon»). Moderniste attentif aux éléments révélateurs de la société traditionnelle (langage, imaginaire social, culture matérielle, figures professionnelles, topographie, objets, etc.), l'historien suit ces sources judiciaires qu'il connaît merveilleusement. Il écrit l'histoire d'un objet qu'il place à la croisée de l'histoire des doctrines et des pratiques médicolégales que génère, dès le XVI ${ }^{\text {mene }}$ siècle en Europe continentale, la procédure inquisitoire (écrite, secrète et basée sur l'aveu et l'expertise matérielle pour fonder la preuve). Sa méthode est convaincante: elle s'inspire de la discipline qu'il étudie en exploitant des «cas médico-légaux» ponctuels pour en tirer des généralités qui montrent les enjeux universels de la médecine-judiciaire dans la constitution des savoirs judiciaires sur le crime.

Dans la pratique, selon Pastore, les routines médico-légales sont marquées par la sociologie professionnelle des experts de l'Ancien Régime, même si parfois la hié-

d'après les lois, registres et arrêts criminels, Paris, 1880; Jean Lecuir, «La médicalisation de la société française dans le deuxième moitié du XVIII ${ }^{\text {ème }}$ siècle en France; aux origines des premiers traités de médecine légale», Annales de Bretagne et des Pays de l'Ouest, 86, 1979, pp. 231-264; Jean-Thomas Levanti, L'évolution de la médecine légale à travers les âges, Bordeaux, 1951; Juan Manuel, Jimenez Munoz, Historia legislativa del cuerpo de medicos forenses, Valladolid, 1974. 
rarchie des compétences née de cette sociologie s'estompe au profit du savoir-faire. Assermentés, «empiriques», barbiers, matrones, chirurgiens et médecins examinaient, selon leurs compétences et selon une hiérarchie socio-professionnelle contraignante, le corps violenté. En cela, ils aident le juge (sommairement appuyé sur des Édits ou des ordonnances en matières criminelles) à qualifier un crime de sang, à mesurer la gravité d'un attentat sexuel ou encore à prouver le caractère accidentel ou volontaire d'une mort violente (accident, noyade, assassinat, suicide, etc.). Très schématiquement: aux matrones et accoucheuses illettrées revenaient la "visite» manuelle des femmes violées, l'attestation de la virginité des «débauchées» ou le contrôle des naissances qu'elles doivent signaler aux autorités. Aux barbiers et chirurgiens «empiriques», incombaient la qualification «clinique» des blessures à armes blanches, le problème complexe de la «percée» des cadavres, ainsi que la «levée des corps» (noyade, accident, homicide, mort suspecte, suicide, etc.). Il n'était pas rare non plus de voir un chirurgien «visitant» le corps dénudé et rasé d'une femme accusée de «maleficium» pour débusquer avec une aiguille la marque satanique dont les caractéristiques «surnaturelles» (insensibilité, absence de saignement) prouvaient le pacte charnel avec le démon. Finalement, les médecins, méprisant les «basses cuvres» (toucher et ouvrir le cadavre) et suivant à la lettre la médecine des Anciens, accaparaient les diagnostics «savants» (empoisonnements), les expertises relatives aux blessures faites par armes à feu, l'avis moral sur la «mélancolie» d'un suicidaire. Spécialistes du corps saisi par la justice, ils pouvaient participer aussi aux séances de torture judiciaire pour guider les juges dans l'administration de la «question $»^{4}$.

En montrant le rôle formidable qu'avaient les experts dans la construction d'un savoir «objectif» et naturaliste des catégories «immorales» de l'anomie sociale dans la société traditionnelle (pp. 25-30) et en documentant les conflits ou les collaborations professionnelles entre chirurgiens et médecins (grosso modo médecine empirique versus médecine savante), Alessandro Pastore construit, de manière comparative, plusieurs objets pour éclairer l'histoire de l'expertise médico-légale. Une expertise qui concerne tout d'abord les juges des tribunaux italiens réglés par les normes de la procédure inquisitoire. Place du corps dans la pénalité de l'Ancien Régime (pp. 30-36), alliance institutionnelle entre le «bourreau» et le chirurgien (pp. 37-32), recherche du stigmate diabolique sur le corps de la sorcière (pp. 43-49), contrôle «moral» et «hygiéniste» de la sexualité et des pratiques médico-judiciaires qui qualifient les transgressions - épreuve de virginité, accouchement secret, avortement, infanticide, etc. - (pp. 49-60;129-148), diagnostic complexe en cas de crime d'empoisonnement (pp. 93-103), ou encore hiérarchie socio-professionnelle et compétitions entre barbiers, chirurgiens et médecins dont les compétences médico-légales (qui touche le corps? qui en effectue la «levée»? qui ouvre le cadavre?, etc.) sont souvent réglementées par le tribunal du «Torrone» à Bologne (pp. 105-127), prise en charge du corps violenté des femmes par l'accoucheuse (pp. 129-148), alliance entre tribunaux et «personnel sanitaire» à Venise (pp. 149-173), dénonciation des crimes de sang par les barbiers de Vérone ou les praticiens de Lucca (pp. 175-189; 191-209), voire impact et enjeux sociaux des expertises médico-légales dans la Lombardie suisse (pp. 211-238).

4 Alessandro Pastore, «Médecine légale et pratique de la torture en Italie au XVIIIme siècle», in Porret, M. (ed.), Beccaria et la culture juridique des Lumières, Genève, Droz, 1997, pp. 287-306. 
Finalement, Alessandro Pastore nous fait comprendre que les légistes contemporains affinent les routines et les usages de la «médecine judiciaire» des chirurgiens et des médecins de l'Ancien Régime. En plaçant la problématique médico-légale de son ouvrage dans la genèse de cette «certitude» normative qui était cruciale sous le régime arbitraire des délits et des peines, il nous donne à lire une substantielle monographie qui renouvelle l'histoire de la justice. La qualification du crime et l'application des peines à l'époque moderne dépendent aussi de la manière dont le pénal a scellé une alliance avec le médical. Les légistes offrent à la justice un savoir matérialiste pour mesurer, idéalement, la «dangerosité» d'un crime ou d'un délinquant selon la pathologie du corps violenté, alors que l'irresponsabilité criminelle est jaugée selon l'étendue de l'aliénation mentale pensée en des catégories morales.

La médecine légale triomphe dès le début du XIX ${ }^{\text {ème }}$ siècle comme la médecine de l'anomie sociale, comme une science positive, «objective» et «rationnelle» autour du slogan «Justice, Médecine et Vérité» pour «prouver» le crime et ses circonstances. Dans un univers laïcisé, le succès des experts du crime de Frédéric Chauvaud est préparé par les légistes de l'Ancien Régime d'Alessandro Pastore qui, dans un monde peu désenchanté, ont mis en place un horizon d'attente rendant possible, après le développement rapide de la médecine légale des Lumières ${ }^{5}$, l'avènement institutionnel et juridique de la médecine légale moderne. Le terme est d'ailleurs un néologisme en français dès les années 1770. Au XVIİ̀me siècle, les légistes d'Italie du nord ressemblent à ceux de Paris du «grand XIX ${ }^{\text {eme }}$ siècle »: en rédigeant des expertises corporelles ou mentales, ils apportent aux juges une sémiologie naturaliste du crime qui idéalement en limite la qualification morale.

La force du beau livre d'Alessandro Pastore réside dans la manière dont il conduit le lecteur à repenser l'histoire du pénal et du médico-légal dans la continuité d'une problématique de la qualification criminelle qui brise les ruptures classiques entre l'Ancien Régime et l'époque contemporaine (arbitraire versus légalité). Hier, comme aujourd'hui, avec des moyens différents, le légiste ne cesse de renforcer son «alliance " avec l'institution pénale qui le sollicite pour «objectiver» les preuves du crime et contribuer aussi à mettre en place un bien juridique moderne qui fondera l'État de droit: l'intégrité de l'individu face à la justice. Telles sont, dans la longue durée de l'histoire des hommes et des délits, les conséquences de la révolution inquisitoriale des XIV ${ }^{\text {eme }}$ et $X V^{\text {ème }}$ siècles. Telle est l'intelligence de l'enquête conduite à son terme par Alessandro Pastore. Elle trouve écho dans le beau livre de Frédéric Chauvaud pour tout lecteur sachant penser l'histoire des normes médicolégales dans la continuité culturelle des pratiques judiciaires et pénales en Europe moderne et contemporaine.

\section{Michel Porret \\ Université de Genève \\ Faculté des Lettres \\ Département d'Histoire Générale \\ Rue Saint-Ours, 5 \\ 1205 Genève - Suisse \\ porret@uni2a.unige.ch}

s Porret, M., «Crimes et châtiments: l'œil du médecin-légiste», XVII ème siècle, 30, Bilan et perspective de la recherche, 1998, pp. 37-50. 